OPEN ACCESS

Edited by:

Andreas Becker,

Universitätsklinikum

Hamburg-Eppendorf, Germany

Reviewed by:

Philipp Mandel,

Universitätsklinikum Hamburg-Eppendorf, Germany

Katharina Boehm,

Universitätsmedizin Mainz, Germany

*Correspondence:

Evanguelos Xylinas

evanguelosxylinas@hotmail.com

Specialty section:

This article was submitted to

Genitourinary Surgery,

a section of the journal

Frontiers in Surgery

Received: 10 October 2017

Accepted: 28 August 2018

Published: 19 September 2018

Citation:

Nguyen T-T, Huillard O, Dabi Y, Anract J, Sibony M, Zerbib $M$ and

Xylinas E (2018) Neoadjuvant

Chemotherapy in Patients With Muscle-Invasive Bladder Cancer and Its Impact on Surgical Morbidity and Oncological Outcomes: A Real-World

Experience. Front. Surg. 5:58.

doi: 10.3389/fsurg.2018.00058

\section{Neoadjuvant Chemotherapy in Patients With Muscle-Invasive Bladder Cancer and Its Impact on Surgical Morbidity and Oncological Outcomes: A Real-World Experience}

\author{
Thanh-Tuan Nguyen ${ }^{1,2}$, Olivier Huillard ${ }^{1}$, Yohann Dabi ${ }^{1}$, Julien Anract ${ }^{1}$, Mathilde Sibony ${ }^{1}$, \\ Marc Zerbib ${ }^{1}$ and Evanguelos Xylinas ${ }^{1 *}$ \\ ${ }^{1}$ Department of Urology and Oncology, Cochin Hospital, Assistance Publique-Hôpitaux de Paris, Paris Descartes University, \\ Paris, France, ${ }^{2}$ Urology Department, University of Medicine and Pharmacy at Ho Chi Minh City, Cho Ray Hospital, Ho Chi \\ Minh City, Vietnam
}

Objectives: The purpose of this study was to investigate the impact of neoadjuvant chemotherapy (NAC) on perioperative morbidity and on oncological outcomes according to the type of chemotherapy regimen administered to patients with muscle-invasive bladder cancer (MIBC) who subsequently underwent radical cystectomy (RC).

Methods: Data were collected retrospectively on 40 patients with bladder urothelial carcinoma who had at least two cycles of NAC, followed by RC, from 2011 to 2015 at our institution. The outcomes evaluated were NAC toxicity, perioperative complications, cancer-specific, and overall survival.

Results: Among these cases, 23 patients (57.5\%) received methotrexate, vinblastine, doxorubicin and cisplatin (MVAC), 4 patients (10\%) received gemcitabine and cisplatin (GC), and 13 patients (32.5\%) received other regimes. The early and late postoperative complication rates were $35 \%$ and $12.5 \%$. Regarding toxicity, $85 \%$ of patients had at least one side effect of NAC, but only $21.7 \%$ discontinued therapy in the MVAC group. The pathological complete response $(\mathrm{pCR})$ rates for cisplatin-based regimens (MVAC and GC) and other regimens were 44.4 and $15.4 \%$, respectively, $(p=0.09)$. The pathological partial response (pPR) rates for cisplatin-based regimens and other regimens were 66.7 and $15.4 \%$, respectively, $(p=0.002)$. Patients treated with a cisplatin-based chemotherapy regimen had longer overall survival than those treated with other regimen (median 38.1 vs. 18.4 months, $p=0.01$ ).

Conclusions: NAC administration was not associated with high toxicity or surgical morbidity. The pathological response rates and survival outcomes in the cisplatin-based regimens were higher than with those with non-cisplatin-based regimens. These data support the use, in patients elective to a neoadjuvant setting prior to $\mathrm{RC}$ for $\mathrm{MBIC}$, of a cisplatin-based regimen.

Keywords: bladder cancer, cystectomy, morbidity, neoadjuvant chemotherapy, survival 


\section{INTRODUCTION}

Muscle-invasive bladder cancer (MIBC) is an aggressive disease with a high-risk of early metastasis and cancerspecific mortality. The gold standard treatment of MIBC is radical cystectomy (RC) in conjunction with concomitant bilateral pelvic lymphadenectomy (1). The prognosis of these patients depends mainly on the histologically determined pathological stage of the radical cystectomy specimen (pT stage) and on the presence of lymph node metastases ( $\mathrm{pN}$ status). Without administration of chemotherapy, $90.5 \%$ of MIBC patients undergoing radical cystectomy have a 10 -year cancerspecific free survival (CSS) if the $\mathrm{pT}$ stage is $\mathrm{pT} 0 / \mathrm{a} / \mathrm{is} / 1 \mathrm{pN} 0$ (nonresidual disease or non-muscle invasive residual disease). The 10 -year CSS decreases to $67 \%$ if this stage is pT2 pN0 (muscle invasive confined disease). Only $16.7 \%$ of patients with nodal metastasis at the time of RC have 10-year cancer-specific free survival, independent of the primary tumor stage (2).

Due to the development and implementation of neoadjuvant chemotherapy (NAC) prior to radical cystectomy, the prognosis for MIBC patients undergoing radical cystectomy has improved $(3,4)$. The goals of neoadjuvant chemotherapy administration are to (1) eradicate the micrometastases, (2) avoid the release and implantation of malignant cells during cystectomy, and (3) extend the survival of these patients.

In the Southwest Oncology Group (SWOG) Intergroup study, RC alone was compared with three cycles of neoadjuvant MVAC (methotrexate, vinblastine, doxorubicin, and cisplatin), followed by radical cystectomy (3). This randomized trial showed that the median survival duration was 77 months in patients with combination treatment, and 46 months in patients with upfront RC alone (3). In terms of overall survival (OS), the group combination therapy recipients had better outcomes (5year OS $=57$ vs. $43 \%, p<0.05$ ) (3). In addition, the rate of stage pT0 disease in the radical cystectomy specimens was significantly higher among the patients who received MVAC combination (3). In the International Collaboration of Trialists study, 976 patients with tumor stage cT2-4N0 were randomized into two groups and underwent either three cycles of neoadjuvant cisplatin, methotrexate, vinblastine (CMV), or no neoadjuvant therapy following local therapy alone. Local therapy comprised either radiotherapy, radical cystectomy, or a combination of lowdose radiotherapy and radical cystectomy (5). This randomized prospective trial showed that CMV combination allowed a $16 \%$ reduction in the risk of death. Following NAC, pathological complete response was 32.5 vs. $12.3 \%$ with radical cystectomy alone (5). Based on these data, cisplatin-based neoadjuvant

\footnotetext{
Abbreviations: CFS, cancer-free survival; DSS, disease specific survival; GC, gemcitabine and cisplatin; GON, gemcitabine, oxaliplatine, and navelbine; GEMOX, gemcitabine and oxaliplatin; HR, hazard ratios; IQR, interquartile range; MIBC, Muscle-invasive bladder cancer; MVAC, methotrexate, vinblastine, doxorubicin and cisplatin; ACG, nab-paclitaxel, carboplatin and gemcitabine; NAC, neoadjuvant chemotherapy; OS, overall survival; pCR, pathological complete response; $\mathrm{PPR}$, pathological partial response; $\mathrm{RC}$, radical cystectomy; SWOG, Southwest Oncology Group; TURBT, transurethral resection of bladder tumor.
}

chemotherapy has demonstrated an improvement in OS in MIBC and has reached a grade recommendation $(1,3-5)$.

However, NAC is rarely administrated in daily practice. In the United States, an analysis of the National Cancer Data Base showed that only $16 \%$ of patients with MIBC underwent NAC (6). In Western and Central Europe, the rate of NAC administration was also low, with only $12 \%$ of patients with MIBC undergoing cystectomy having received NAC (7). The reasons for this low implementation rate of NAC include, among general toxicity concerns, increased perioperative complications, potentially ineffective chemotherapy, and delay in curative-intent surgery. Therefore, the management of MIBC remains controversial, with debate existing over neoadjuvant chemotherapy plus radical cystectomy, vs. upfront surgery alone.

Therefore, the present study retrospectively analyzed the impact on surgical morbidity and on the oncological outcomes of MIBC patients at our institution who underwent radical cystectomy with prior neoadjuvant chemotherapy.

\section{METHODS}

\section{Patients}

The study was designed as a retrospective investigation, in which 50 consecutive patients treated with neoadjuvant chemotherapy before undergoing radical cystectomy with bilateral pelvic lymphadenectomy at Cochin hospital from July 2011 to July 2015 were included. Patients who had indication of radical cystectomy (cT2-4aNxM0 or high risk T1) and received at least two cycles of NAC followed by RC were selected for further analysis. Patients with any other histological type that pure urothelial carcinoma or mixed histology with squamous and/or glandular differentiation were excluded. Our final cohort included 40 patients [10 were excluded because of $<2$ cycles of chemotherapy $(n=8)$ and because of lost to follow-up $(n=2)]$.

\section{Pathological Analyses}

All transurethral resection and cystectomy specimens underwent pathological review by specialist genitourinary pathologists at our institution. Clinical staging was based on transurethral resection of bladder tumor (TURBT) and preoperative imaging. Postoperative pathological analyses included tumor classification, presence of carcinoma in situ, surgical margin status, number of lymph nodes and TNM staging according to the 2010 American Joint Committee on cancer classification. One of our primary outcomes was pathological response to neoadjuvant chemotherapy. Complete pathological response was defined as pT0N0 stage on the radical cystectomy specimen and partial pathological response was defined as pT0N0, pTaN0, pTisN0, or pT1N0.

\section{Therapeutic Sequence}

All patients were included in a multidisciplinary tumor board at which NAC administration was decided. The choice of chemotherapy regimen was then made at the referent oncologist's discretion based on patient's clinical features. The NAC regimens included MVAC, GC (gemcitabine and cisplatin) or Other. Typical regimen of MVAC chemotherapy 
was four cycles at 28-day intervals over 16 weeks with methotrexate $30 \mathrm{mg} / \mathrm{m}^{2}$ (days 1, 15, and 22), vinblastine 3 $\mathrm{mg} / \mathrm{m}^{2}$ (days 2, 15, and 22), doxorubicin $30 \mathrm{mg} / \mathrm{m}^{2}$ (day 2), and cisplatin $70 \mathrm{mg} / \mathrm{m}^{2}$ (day 2). Patients in the GC group received typically four cycles at 21-day interval over 12 weeks with gemcitabine $1,000 \mathrm{mg} / \mathrm{m}^{2}$ (days 1,8 , and 15) and cisplatin $70 \mathrm{mg} / \mathrm{m}^{2}$ (day 2). The Other group consisted of patients receiving gemcitabine with carboplatin, GON (gemcitabine, oxaliplatine, and navelbine) or GEMOX (gemcitabine and oxaliplatin). During NAC administration, the dose of chemotherapy was adjusted based on creatinine and chemotherapy toxicities. NAC data included types of regimen, number of cycles, change of NAC dose, and toxicities of chemotherapy.
Following neoadjuvant chemotherapy, radical cystectomy with standard pelvic lymphadenectomy was performed on all patients. The operative variables included the time between diagnosis and surgery, type of urinary diversion and surgical morbidity. During the follow-up, we analyzed the perioperative complications of RC according to the Clavien classification system. Early and late complications were defined as a postoperative complication within 30 days and from 30 to 90 days, respectively.

After surgery, patients were seen every 3 months during the first year, every 6 months the year after and every year after that. Radiological exams (CT abdomen /pelvis with intravenous contrast) were practiced every year or at any moment if the physician suspected disease recurrence. Disease recurrence was

TABLE 1 | Clinical characteristics, operative features, and pathologic outcomes of patients receiving neoadjuvant chemotherapy.

\begin{tabular}{|c|c|c|c|c|}
\hline NAC regimen & MVAC $(n=23)$ & $\mathrm{GC}(n=4)$ & Other $(n=13)$ & Total \\
\hline Age at RC, year, median (IQR) & $62(56-70)$ & $70(65-74)$ & $68(65-75)$ & $65(61-71)$ \\
\hline Male, $n(\%)$ & $20(87)$ & $3(75)$ & $9(69.2)$ & $32(80)$ \\
\hline \multicolumn{5}{|l|}{ CLINICAL STAGE AT PRESENTATION, $n(\%)$} \\
\hline T1 & $2(8.7)$ & 0 & $1(7.7)$ & $3(7.5)$ \\
\hline T2 & $20(87)$ & $3(75)$ & $8(61.5)$ & $31(77.5)$ \\
\hline T3 & $1(4.3)$ & $1(25)$ & $2(15.4)$ & $4(10)$ \\
\hline T4a & 0 & 0 & $2(15.4)$ & $2(5)$ \\
\hline NO & $17(73.9)$ & $4(100)$ & $6(46.2)$ & $27(67.5)$ \\
\hline N1 & $2(8.7)$ & 0 & $1(7.7)$ & $3(7.5)$ \\
\hline N2 & $4(17.4)$ & 0 & $6(46.2)$ & $10(25)$ \\
\hline Associated CIS, $n$ (\%) & $3(13)$ & 0 & 0 & $3(7.5)$ \\
\hline \multicolumn{5}{|l|}{ HISTOLOGIC GRADE OF TUMOR } \\
\hline 2 & $1(4.3)$ & 0 & 0 & $1(2.5)$ \\
\hline 3 & $22(95.7)$ & $4(100)$ & $13(100)$ & $39(97.5)$ \\
\hline \multicolumn{5}{|l|}{ PRESENCE OF HYDRONEPHROSIS, $\boldsymbol{n}(\%)$} \\
\hline No hydronephrosis & $19(82.6)$ & $3(75)$ & $6(46.2)$ & $28(70)$ \\
\hline Unilateral hydronephrosis & $3(13)$ & $1(25)$ & $6(46.2)$ & $10(25)$ \\
\hline Bilateral hydronephrosis & $1(4.3)$ & 0 & $1(7.7)$ & $2(5)$ \\
\hline $\begin{array}{l}\text { Median of the time between last TURBT and } \\
\text { cystectomy, days, (IQR) }\end{array}$ & $133(111-154)$ & $161(136-265)$ & $156(106-176)$ & $142.5(112-163)$ \\
\hline \multicolumn{5}{|c|}{ NUMBER OF CYCLES OF NAC COMPLETED, $\boldsymbol{n}(\%)$} \\
\hline 2 & $2(8.7)$ & $1(25)$ & 0 & $3(7.5)$ \\
\hline 3 & $3(13)$ & $1(25)$ & $1(7.7)$ & $5(12.5)$ \\
\hline 4 & $11(47.8)$ & $2(50)$ & $9(69.2)$ & $22(55)$ \\
\hline$\geq 5$ & $7(30.4)$ & 0 & $3(23.1)$ & $10(25)$ \\
\hline $\begin{array}{l}\text { Change of NAC dose or regime during } \\
\text { chemotherapy, } n(\%)\end{array}$ & $3(13)$ & $2(50)$ & $2(15.4)$ & 7 (17.5) \\
\hline \multicolumn{5}{|l|}{ TYPE OF URINARY DIVERSION, $\boldsymbol{n}(\%)$} \\
\hline Ileal conduit & 9 (39.1) & $2(50)$ & $10(76.9)$ & $21(52.5)$ \\
\hline Orthotopic neobladder & $14(60.9)$ & $2(50)$ & $3(23.1)$ & $19(47.5)$ \\
\hline \multicolumn{5}{|l|}{ PATHOLOGIC OUTCOME } \\
\hline pTONO & $12(52.2)$ & 0 & $2(15.4)$ & $14(35)$ \\
\hline$\leq \mathrm{pT} 1 \mathrm{NO}$ & $17(73.9)$ & $1(25)$ & $2(15.4)$ & $20(50)$ \\
\hline Positives nodes, median (range) & $0(0-1)$ & $0.5(0-1)$ & $0(0-3)$ & $0(0-3)$ \\
\hline Positive surgical margins, $n$ (\%) & $1(4.3)$ & 0 & $2(15.4)$ & $3(7.5)$ \\
\hline Associated CIS post-operation, $n(\%)$ & $4(17.4)$ & 0 & $2(15.4)$ & $6(15)$ \\
\hline
\end{tabular}


defined as tumor relapse in the operative field, regional lymph nodes and/or distant metastases. Death's cause was determined using patient's death certificate. Death within 30 days after surgery was censored for specific survival analysis.

The oncological outcomes included pathological response, OS and CSS.

\section{Statistical Analyses}

Variables collected in the database included patient demographics, clinical staging, NAC regimen features, radical cystectomy procedure data, pathological features, surgical morbidity, postoperative morbidity, and oncological outcomes. For variables with non-normal distribution, data were presented as median and interquartile range (IQR). Means and medians were reported for continuous variables. Proportions and frequencies were reported for categorical variables. Means were compared by using the Student's Ttest. For variables with non-normal distribution, the two groups were compared by using the Mann-Whitney $U$ test. When there were more than three groups, the KruskalWallis test was used. Proportions were compared by using Chi-squared tests with continuity correction of Fisher's exact test when appropriate. The Kaplan-Meier method and

TABLE 2 | Toxicity of neoadjuvant chemotherapy.

\begin{tabular}{lcccc}
\hline Toxicity, $\boldsymbol{n}(\%)$ & Grade $\mathbf{1}$ & Grade 2 & Grade 3 & Grade 4 \\
\hline Neutropenia & $3(7.5)$ & $3(7.5)$ & $2(5)$ & $1(2.5)$ \\
Anemia & $5(12.5)$ & $1(2.5)$ & $1(2.5)$ & 0 \\
Thrombocytopenia & $6(15)$ & $1(2.5)$ & $2(5)$ & 0 \\
Gastrointestinal effects (nausea, & $12(30)$ & $5(12.5)$ & $2(5.0)$ & 0 \\
vomiting, diarrhea, or constipation) & & & & \\
Renal insufficiency & $5(12.5)$ & 0 & 0 & 0 \\
Mucositis & $3(7.5)$ & $2(5)$ & 0 & 0 \\
Neuropathy & $4(10)$ & $1(2.5)$ & 0 & 0 \\
Fatigue or malaise & $9(22.5)$ & $3(7.5)$ & $1(2.5)$ & 0 \\
Infection & $2(5)$ & $1(2.5)$ & 0 & 0
\end{tabular}

multivariable Cox proportional hazards regression model were used to analyze occurrence of cancer-specific and overall mortality. The analyses were performed with the SPSS software version 20.0 (IBM SPSS Statistics; IBM Corp, Armonk, NY, USA).

\section{RESULTS}

\section{Patient Demographics and Clinical Features}

The patient characteristics, the surgical features and pathological outcomes according to the type of NAC are shown in Table 1. The median age of the population was 65 (IQR, 61-71 years) and the ratio of males to females was 4:1. In the Other regimens group, eight patients received GEMOX, four received GON and one patient received carboplatin with gemcitabine. There were no differences between the different NAC groups with regards to gender, clinical stage of tumor at diagnosis, associated CIS, histological grade of tumor, or hydronephrosis at presentation and time between last TURBT and cystectomy. However, there was a significant difference in age among the three groups $(p=0.044)$.

\section{Neoadjuvant Chemotherapy}

NAC toxicities are displayed in Table 2. In this study, $85 \%$ of patients suffered at least one adverse effect. The proportions of incomplete chemotherapy due to toxicity (less than four cycles of NAC) were $21.7 \%$ for MVAC, 50\% for GC, and 7.7\% for the Other regimens $(7.7 \%)(p=0.172)$.

\section{Surgical Morbidity}

Early and late morbidity data for patients treated with NAC prior to RC are presented in Table 3. This study showed that 35\% of patients had at least one early complication after RC followed by one late complication in $12.5 \%$ cases. Overall minor (grade 1-2) and major (grade 3-5) complications rates were 66.7 and $33.3 \%$, respectively. In the group of late complication, two patients died secondly to an intestinal obstruction and to a septicemia.

TABLE 3 | Early and late Postoperative complications after neoadjuvant chemotherapy and radical cystectomy.

\begin{tabular}{|c|c|c|c|c|c|c|c|c|c|c|}
\hline \multirow[t]{2}{*}{ Complication, $\boldsymbol{n}$ (\%) } & \multicolumn{5}{|c|}{ Early complication ( $\leq 30$ days) } & \multicolumn{5}{|c|}{ Late complication ( $>30$ days and $\leq 90$ days) } \\
\hline & Grade 1 & Grade 2 & Grade 3 & Grade 4 & Grade 5 & Grade 1 & Grade 2 & Grade 3 & Grade 4 & Grade 5 \\
\hline Wound infection & 0 & 0 & 0 & 0 & 0 & 0 & 0 & 0 & 0 & 0 \\
\hline Urinary infection & 0 & $8(20)$ & $1(2.5)$ & $1(2.5)$ & 0 & 0 & $1(2.5)$ & 0 & 0 & $1(2.5)$ \\
\hline Hemorrhage & 0 & 0 & 0 & $1(2.5)$ & 0 & 0 & 0 & 0 & 0 & $1(2.5)$ \\
\hline Urinary leakage & 0 & $1(2.5)$ & 0 & 0 & 0 & 0 & 0 & 0 & 0 & 0 \\
\hline lleus & 0 & $1(2.5)$ & $1(2.5)$ & 0 & 0 & 0 & 0 & 0 & 0 & 0 \\
\hline $\begin{array}{l}\text { Renal effects (acute renal } \\
\text { insufficiency) }\end{array}$ & 0 & $3(7.5)$ & 0 & 0 & 0 & 0 & 0 & $2(5)$ & 0 & 0 \\
\hline Lymphoceles & $1(2.5)$ & 0 & 0 & 0 & 0 & 0 & 0 & 0 & 0 & 0 \\
\hline $\begin{array}{l}\text { Thrombosis or embolism (pulmonary } \\
\text { embolism, deep venous thrombosis) }\end{array}$ & 0 & $1(2.5)$ & 0 & 0 & 0 & 0 & 0 & 0 & 0 & 0 \\
\hline Total & $1(2.5)$ & $14(35)$ & $2(5)$ & $2(5)$ & 0 & 0 & $1(2.5)$ & $2(5)$ & 0 & $2(5)$ \\
\hline
\end{tabular}


TABLE 4 | Correlation of clinical stage and final pathologic stage stratified by NAC.

\begin{tabular}{|c|c|c|c|c|c|c|c|}
\hline & pTONO (pCR) & pTis/pTa/pT1NO & $\leq$ pT1NO (pPR) & pT2NO & pT3-4NO & pTanyN1-3 & Total \\
\hline \multicolumn{8}{|c|}{ MVAC NAC, $n(\%)$} \\
\hline cT1 & 0 & $1(50)$ & $1(50)$ & 0 & $1(50)$ & 0 & 2 \\
\hline cT2 & $12(60)$ & $3(15)$ & $15(75)$ & 1 (5) & $3(15)$ & 1 (5) & 20 \\
\hline сT3 & 0 & $1(100)$ & $1(100)$ & 0 & 0 & 0 & 1 \\
\hline cT4 & 0 & 0 & 0 & 0 & 0 & 0 & 0 \\
\hline cT1 & 0 & 0 & 0 & 0 & 0 & 0 & 0 \\
\hline cT2 & 0 & 1 (33.3) & $1(33.3)$ & 0 & 1 (33.3) & $1(33.3)$ & 3 \\
\hline cT3 & 0 & 0 & 0 & 0 & 0 & $1(100)$ & 1 \\
\hline cT4 & 0 & 0 & 0 & 0 & 0 & 0 & 0 \\
\hline Total & 0 & $1(25)$ & $1(25)$ & 0 & $1(25)$ & $2(50)$ & 4 \\
\hline cT3 & 0 & 0 & 0 & 0 & $2(100)$ & 0 & 2 \\
\hline cT4 & 0 & 0 & 0 & 0 & 0 & $2(100)$ & 2 \\
\hline Total & $2(15.4)$ & 0 & $2(15.4)$ & $1(7.7)$ & $5(38.5)$ & $5(38.5)$ & 13 \\
\hline
\end{tabular}

GC, gemcitabine and cisplatin; MVAC, methotrexate, vinblastine, doxorubicin, cisplatin; NAC, neoadjuvant chemotherapy; pCR, pathologic complete response; pPR, pathologic partial response.
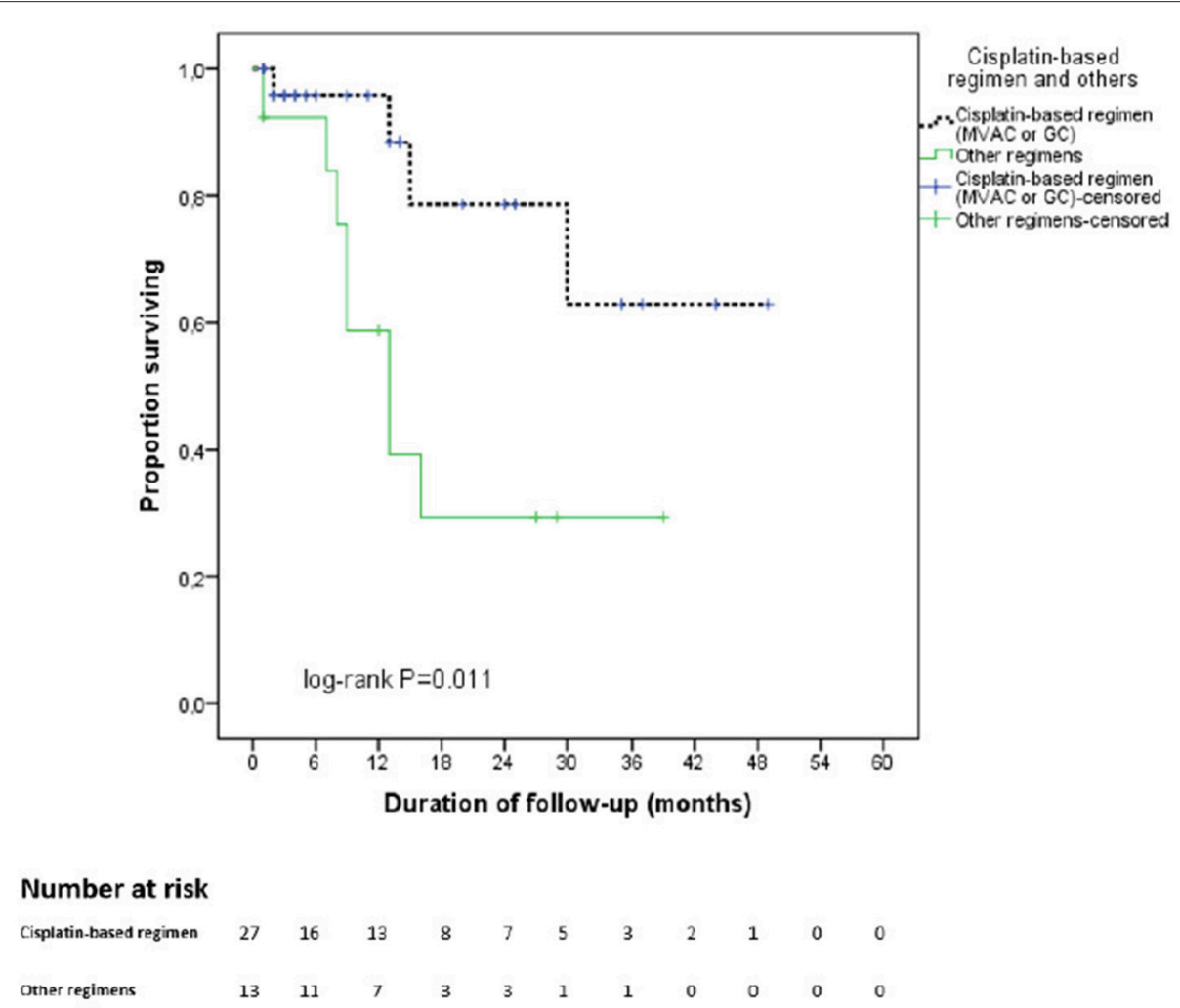

FIGURE 1 | Kaplan Meier curve for overall survival stratified by type of neoadjuvant chemotherapy regimen. In black: cisplatin-based chemotherapy regimen. In green: other regimens. cnoinall survival elected in a t benefitsing prior to RC for MBIC ts with lower performance status that were not selected in at $\mathrm{cn}$. 


\section{Pathological Outcomes}

The pathological outcomes stratified by the NAC regimens are displayed in Table 4. The pCR rates were significantly higher (44.4\%) for the cisplatin-based regimens (MVAC and GC) vs. $15.4 \%$ for the other regimens $(p=0.09)$. The $p P R$ rates for the cisplatin-based regimens and other regimens were 66.7 and $15.4 \%$, respectively, $(p=0.002)$.

\section{Survival Outcomes}

In the entire cohort, the median duration of follow-up was 21.5 months and the estimated mean OS time was 30.7 months. Figure 1 displays the Kaplan-Meier curves for OS stratified by NAC regimen. Specifically, patients were divided into cisplatinbased regimens (MVAC or GC) and Other regimens. Patients receiving cisplatin-based regimens had better OS outcomes (38.1 vs. 18.4 months, $p=0.011$ ).

The estimated CSS mean time for the entire cohort was 30.1 months based on Kaplan-Meier method. Figure 2 displays the Kaplan-Meier curves for CSS stratified by NAC regimens. Patients receiving cisplatin-based regimens had better CSS outcomes (37.6 vs. 14.3 months, $p=0.004$ ).

With regards to pathological response, Figure 3 shows the Kaplan-Meier curves for OS of the two groups with and without pathological partial response. The OS of patients with pPR was better than that of the remaining patients (43 vs. 19.5 months, $p=0.012$ ).
In the Cox proportional hazards regression model for overall mortality, age (HR: 1.17 [95\% CI, 1.02-1.33]) and positive surgical margins status (HR: 11.93 [95\% CI, 1.13-125.81]) were significantly associated with overall mortality (Table 5).

\section{DISCUSSION}

One disinclination to the wider administration of NAC derives from the concern of increased postoperative complications after radical cystectomy in patients receiving this regimen. The results of our study showed that morbidity and mortality during early and late period of follow-up (30 and 90 days, respectively) were similar to the reported complications rates of upfront radical cystectomy series (without administration of NAC) (2, 8-12). Previous studies reported that the early complications rates were $19.7-39.5 \%$, the late complications rates were $10.8-19.8 \%$, the early mortality rates were $0.4-3.2 \%$ and finally the late mortality rates were 3 to $9 \%(2,8-12)$. Our results are in line with those of Johnson et al. (13) who showed no increased perioperative complications/surgical morbidity when comparing two groups of patients treated with RC, one with NAC administration and one without (13). Therefore, NAC is not associated with excessive post-operative morbidity and should be recommended as long as it improves the oncological outcomes.

Several studies emphasized that MVAC-based NAC administration improves survival in patients treated with
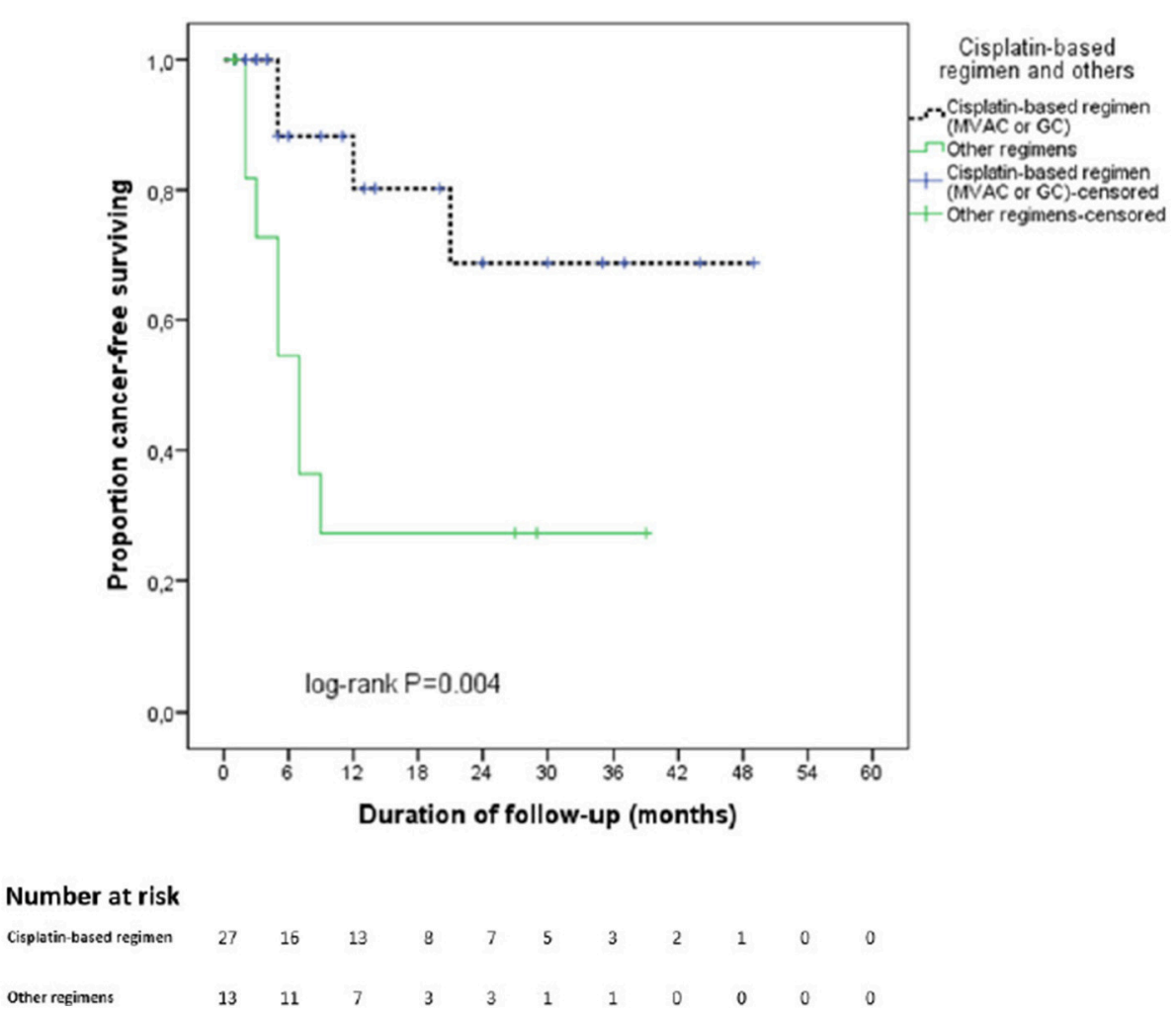

FIGURE 2 | Kaplan Meier curve for cancer specific survival stratified by type of neoadjuvant chemotherapy regimen. In black: cisplatin-based chemotherapy regimen. In green: other regimens. 

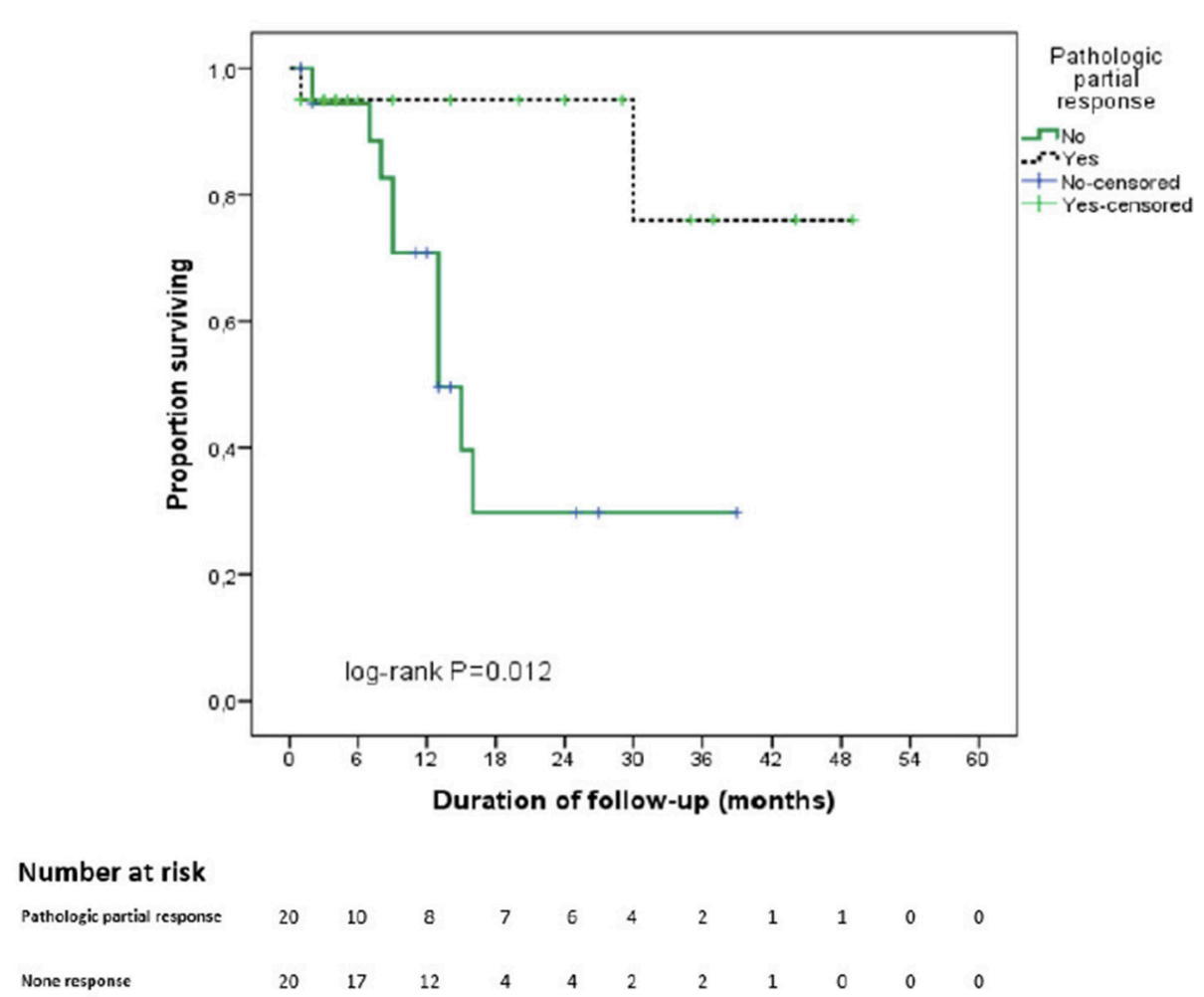

FIGURE 3 | Kaplan Meier curve for overall survival stratified by pathological partial response. In black: partial pathological response on final pathological analysis. In green: absence of partial pathological response on final pathological analysis.

TABLE 5 | Cox regression model assessing factors predicting overall mortality occurrence.

\begin{tabular}{lcc}
\hline Variable & Cox regression analysis & \\
\hline & HR (95\% CI) & $\boldsymbol{p}$-value \\
Age & $1.17(1.02-1.33)$ & $\mathbf{0 . 0 2 4}$ \\
GENDER & 1 & \\
Male & $1.62(0.27-9.81)$ & 0.6 \\
Female & 1 & \\
CHEMOTHERAPY REGIMEN & $3.23(0.22-48.45)$ & 0.4 \\
MVAC & $3.05(0.28-32.95)$ & 0.36 \\
GC & 1 & \\
Other regimens & $11.93(1.13-125.81)$ & $\mathbf{0 . 0 4}$ \\
Surgical margin & & \\
Negative & 1 & 0.65 \\
Positive & $0.61(0.07-5.07)$ & \\
PCR (PTON0) & & \\
No & & \\
Yes & & \\
\hline
\end{tabular}

$P$-value $<0.05$ is significant which means age and positive surgical margins status were significantly associated with overall mortality.

RC (3, 5, 14-16). Moreover, several studies revealed that GC regimens was associated with similar pathological response rates and survival outcomes compared to MVAC regimens (4, 17-19). In this study, we showed that patients receiving cisplatin-based regimens, the pCR and pPR were 44.4 and $66.7 \%$, respectively. These data were higher than previous reported studies where the pCR following NAC varied from 22.7 to $38 \%(3,5,20)$. This difference could be explained by the low proportion of locally-advanced disease before NAC in our cohort compared to previous reported cohorts. In fact, the proportion of cT3-4a in the cisplatin-based group of our series $(7.4 \%)$ was lower for example than in the MVAC group of SWOG trial and the Nordic Urothelial Cancer Group trial (48.6 and $59.1 \%)(3,20)$. In our cohort, similar to a multicenter study published by Zargar et al. (4), the pPR rate of the cisplatinbased regimen was superior significantly to the other regimen group ( 66.7 vs. $15.4 \%, p=0.002$ ) (4). The pCR rates of the cisplatin-based regimens were better than those of the other regimens, although the difference was not statistically significant ( 44.4 vs. $15.4 \%, p=0.09$ ). The pathological response after NAC is strongly associated with overall survival and is one of the most important factors in order to evaluate the efficacy of NAC $(4,20,21)$. Similarly, we found out that OS of the pPR group was higher compared to the rest of our cohort (43 vs. 19.5 months, $p=0.012$ ). The OS was as well higher in the pCR group compared to the rest of our series, although the difference was not statically significant (39.7 vs. 26 months, $p=0.26$ ). These rates are lower than those reported by Grossman et al. in the SWOG study. One possible explanation is that we included patients with lower performance status representing perhaps a more real-life cohort than the patients usually selected in a clinical trial. Previous studies have reported that the patients harboring a pT0 stage at RC had a survival benefit $(22,23)$. In the 
NAC setting, the use of cisplatin-based regimen was a predictor of favorable pathological response which might translates into survival benefits.

Our study harbors important limitations mainly due to its retrospective design. First, the choice of NAC regimen was influenced by many factors such as performance status, renal function, and comorbidity of patients that could also affect the outcomes. Therefore, our series have some imbalances in patient characteristics between the NAC regimen groups. Secondly, our study was also limited by a small sample size that challenges the statistical analyses. Third, we did not assess the information on NAC dose density, growth factor support and performance status. These limitations may impact our estimates of pathological response and survival outcomes. Nonetheless, we could determine the perioperative morbidity and oncological outcomes according NAC regimens. One strength of our study is that we report a real-world practice cohort different from the selected patients usually enrolled in the clinical trials.

In conclusion, our results augment the body of evidence of the previously reported beneficial effects of NAC from a daily

\section{REFERENCES}

1. Alfred Witjes J, Lebret T, Comperat EM, Cowan NC, De Santis M, Bruins HM, et al. Updated 2016 EAU Guidelines on -invasive and metastatic bladder cancer. Eur Urol. (2017) 71:462-75. doi: 10.1016/j.eururo.2016.06.020

2. Hautmann RE, de Petriconi RC, Pfeiffer C, Volkmer BG. Radical cystectomy for urothelial carcinoma of the bladder without neoadjuvant or adjuvant therapy: long-term results in 1100 patients. Eur Urol. (2012) 61:1039-47. doi: 10.1016/j.eururo.2012.02.028

3. Grossman HB, Natale RB, Tangen CM, Speights VO, Vogelzang NJ, Trump DL, et al. Neoadjuvant chemotherapy plus cystectomy compared with cystectomy alone for locally advanced bladder cancer. $N$ Engl J Med. (2003) 349:859-66. doi: 10.1056/NEJMoa022148

4. Zargar H, Espiritu PN, Fairey AS, Mertens LS, Dinney CP, Mir MC, et al. Multicenter assessment of neoadjuvant chemotherapy for muscle-invasive bladder cancer. Eur Urol. (2015) 67:241-9. doi: 10.1016/j.eururo.2014.0 9.007

5. Griffiths G, Hall R, Sylvester R, Raghavan D, Parmar MK. International phase III trial assessing neoadjuvant cisplatin, methotrexate, and vinblastine chemotherapy for muscle-invasive bladder cancer: long-term results of the BA06 30894 trial. J Clin Oncol. (2011) 29:2171-7. doi: 10.1200/JCO.2010.3 2.3139

6. Zaid HB, Patel SG, Stimson C, Resnick MJ, Cookson MS, Barocas DA, et al. Trends in the utilization of neoadjuvant chemotherapy in muscle-invasive bladder cancer: results from the National Cancer Database. Urology (2014) 83:75-80. doi: 10.1016/j.urology.2013.07.072

7. Burger $\mathrm{M}$, Mulders $\mathrm{P}$, Witjes W. Use of neoadjuvant chemotherapy for muscle-invasive bladder cancer is low among major European centres: results of a feasibility questionnaire. Eur Urol. (2012) 61:1070-1. doi: 10.1016/j.eururo.2012.01.039

8. Kim SH, Yu A, Jung JH, Lee YJ, Lee ES. Incidence and risk factors of 30-day early and 90-day late morbidity and mortality of radical cystectomy during a 13-year follow-up: a comparative propensity-score matched analysis of complications between neobladder and ileal conduit. Jpn J Clin Oncol. (2014) 44:677-85. doi: 10.1093/jjco/hyu051

9. Stimson CJ, Chang SS, Barocas DA, Humphrey JE, Patel SG, Clark PE, et al. Early and late perioperative outcomes following radical cystectomy: 90-day readmissions, morbidity and mortality in a contemporary series. J Urol. (2010) 184:1296-300. doi: 10.1016/j.juro.2010.06.007 practice viewpoint. NAC administration was not associated with high toxicity or surgical morbidity. The pathological response rates and survival outcomes in the cisplatin-based regimen groups were higher than those in the non-cisplatin-based regimen groups. These data support the use of the cisplatin-based regimen in the neoadjuvant setting prior to RC for MIBC.

\section{ETHICS STATEMENT}

This study was carried out in accordance with the recommendations of French guidelines, CPP committee with written informed consent from all subjects.

All subjects gave written informed consent in accordance with the Declaration of Helsinki. The protocol was approved by the CPP from Ile de France.

\section{AUTHOR CONTRIBUTIONS}

All authors listed have made substantial, direct, and intellectual contribution to the work and approved it for publication.

10. Ramani VA, Bromage SJ, Clarke NW. A contemporary standard for morbidity and outcome after radical cystectomy. BJU Int. (2009) 104:628-32. doi: 10.1111/j.1464-410X.2009.08506.x

11. Aziz A, May M, Burger M, Palisaar RJ, Trinh QD, Fritsche HM, et al. Prediction of 90-day mortality after radical cystectomy for bladder cancer in a prospective European Multicenter Cohort. Eur Urol. (2014) 66:156-63. doi: 10.1016/j.eururo.2013.12.018

12. Novara G, De Marco V, Aragona M, Boscolo-Berto R, Cavalleri S, Artibani W,et al. Complications and mortality after radical cystectomy for bladder transitional cell cancer. J Urol. (2009) 182:914-21. doi: 10.1016/j.juro.2009.05.032

13. Johnson DC, Nielsen ME, Matthews J, Woods ME, Wallen EM, Pruthi RS, et al. Neoadjuvant chemotherapy for bladder cancer does not increase risk of perioperative morbidity. BJU Int. (2014) 114:221-8. doi: 10.1111/bju.12585

14. Stenzl A, Cowan NC, De Santis M, Kuczyk MA, Merseburger AS, Ribal MJ, et al. Treatment of Muscle-invasive and Metastatic Bladder Cancer: update of the EAU Guidelines. Eur Urol. (2011) 59:1009-18. doi: 10.1016/j.eururo.2011.03.023

15. Advanced Bladder Cancer Meta-analysis Collaboration. Neoadjuvant chemotherapy in invasive bladder cancer: a systematic review and metaanalysis. Lancet (2003) 361:1927-34. doi: 10.1016/S0140-6736(03)13580-5

16. Advanced Bladder Cancer (ABC) Meta-analysis Collaboration. Neoadjuvant chemotherapy in invasive bladder cancer: update of a systematic review and meta-analysis of individual patient data advanced bladder cancer (ABC) meta-analysis collaboration. Eur Urol. (2005) 48:202. doi: 10.1016/j.eururo.2005.04.006

17. Dash A, Pettus JA, Herr HW, Bochner BH, Dalbagni G, Donat SM, et al. A role for neoadjuvant gemcitabine plus cisplatin in muscle-invasive urothelial carcinoma of the bladder. Cancer (2008) 113:2471-7. doi: 10.1002/cncr.23848

18. Fairey AS, Daneshmand S, Quinn D, Dorff T, Dorin R, Lieskovsky $G$, et al. Neoadjuvant chemotherapy with gemcitabine/cisplatin vs. methotrexate/vinblastine/doxorubicin/cisplatin for muscle-invasive urothelial carcinoma of the bladder: a retrospective analysis from the University of Southern California. Urol Oncol. (2013) 31:1737-43. doi: 10.1016/j.urolonc.2012.07.005

19. Pal SK, Ruel NH, Wilson TG, Yuh BE. Retrospective analysis of clinical outcomes with neoadjuvant cisplatin-based regimens for muscleinvasive bladder cancer. Clin Genitourin Cancer (2012) 10:246-50. doi: 10.1016/j.clgc.2012.08.004 
20. Rosenblatt R, Sherif A, Rintala E, Wahlqvist R, Ullén A, Nilsson S, et al. Pathologic downstaging is a surrogate marker for efficacy and increased survival following neoadjuvant chemotherapy and radical cystectomy for muscle-invasive urothelial bladder cancer. Eur Urol. (2012) 61:1229-38. doi: 10.1016/j.eururo.2011.12.010

21. Petrelli F, Coinu A, Cabiddu M, Ghilardi M, Vavassori I, Barni S. Correlation of pathologic complete response with survival after neoadjuvant chemotherapy in bladder cancer treated with cystectomy: a meta-analysis. Eur Urol. (2014) 65:350-7. doi: 10.1016/j.eururo.2013.0 6.049

22. May M, Bastian PJ, Burger M, Bolenz C, Trojan L, Herrmann E, et al. Multicenter evaluation of the prognostic value of pT0 stage after radical cystectomy due to urothelial carcinoma of the bladder. BJU Int. (2011) 108:E278-83. doi: 10.1111/j.1464-410X.2011.10 189.x
23. Tilki D, Svatek RS, Novara G, Seitz M, Godoy G, Karakiewicz PI, et al. Stage pT0 at radical cystectomy confers improved survival: an international study of 4,430 patients. J Urol. (2010) 184:888-94. doi: 10.1016/j.juro.2010.04.081

Conflict of Interest Statement: The authors declare that the research was conducted in the absence of any commercial or financial relationships that could be construed as a potential conflict of interest.

Copyright (๔) 2018 Nguyen, Huillard, Dabi, Anract, Sibony, Zerbib and Xylinas. This is an open-access article distributed under the terms of the Creative Commons Attribution License (CC BY). The use, distribution or reproduction in other forums is permitted, provided the original author(s) and the copyright owner(s) are credited and that the original publication in this journal is cited, in accordance with accepted academic practice. No use, distribution or reproduction is permitted which does not comply with these terms. 